\title{
Nerve blocks and cognitive therapy: A beneficial failure
}

\author{
Harold Merskey DM FRCP \\ Editor-in-Chief, Pain Research \& Management \\ Ellen N Thompson MBBS FRCPC \\ Clinical Assistant Professor, \\ University of Ottawa
}

$T^{n-10}$ he trial by Gale et al (pages 185-189) is a valuable example of a negative result - the sort of finding that is published less often than a positive one. But, we can learn from the failure, both in understanding correct treatment and in planning future trials. Patients were recruited in a clinic where repeated treatment by nerve blocks is used as a palliative measure for chronic pain of all types. They were then offered the choice of entering a group with cognitive behavioural therapy or continuing with nerve blocks. For ethical reasons, patients could freely leave either branch of the trial without prejudice. At the onset, one of 34 patients in the nerve-block group left, while 12 departed from the cognitive therapy group. All 33 patients remaining in the nerve block group completed the eight-week trial, while only four of 34 patients completed it in the cognitive therapy group.

Three conclusions followed. Too few patients remained in the cognitive therapy group to permit an intergroup comparison of efficacy. Those who conduct any future trial should beware of using patients who have established an existing preference; and there may be a small subgroup that will respond to cognitive behavioural therapy and should be recognized, treated and identified if possible for further attention.

Meanwhile, nerve-block treatment continues to present a conundrum. Patients benefit for longer periods of time than the duration of the anesthetic used. This phenomenon has been documented in the literature, and is regularly observed by clinicians, particularly those with an anesthesia background. Arner et al (1) reported that two-thirds of 38 patients with neuralgic pain experienced up to six days of relief from local anesthetic blocks of the affected nerve. But despite evidence that the practice of local anesthetic blocks for chronic pain is widespread, few trials have been published. While the current study may have certain limitations, it is necessary to continue this line of work.

The mechanisms by which local anesthetics abolish chronic pain for several days when they are effective for a maximum of $4 \mathrm{~h}$ if used for acute or 'physiological' pain, are not known. Several theories have been suggested. In an essay on the future of local anesthetics, Wall (2) lists several. The sympathetic nervous system has been implicated (3) and in a series of articles in Pain Reviews, McCormack $(4,5)$ speculated that such blocks cause temporary abolition of spontaneous ectopic discharges, resulting in abolition of dynamically maintained central hyperexcitability, as well as reinforcing endogenous G-protein-coupled receptor inhibition of n-type voltage-sensitive calcium channels. Finally, the new data on glial activation in pathological pain (6) may cast doubt on the utility of cognitive behavioural therapy and other psychological interventions, while lending new legitimacy to local anesthetic block procedures, 
explaining the findings in the Gale et al (pages 185-189) study. The work of Linda Watkins et al (6) shows that spinal cord glia can be activated in response to a variety of stimuli, both tissue injury and infections, bacterial and viral. The activated glia produce a number of proinflammatory cytokines associated with central sensitization. This activation spreads from cell to cell across 'gap junctions', following no particular neuronal pathways or anatomical boundaries.

Up to the present, such pain has been termed 'medically unexplained' (formerly 'psychogenic, 'somatizing' or even 'hysterical'). It was thought that maladaptive psychological processes were primarily responsible for causing regional pain, and it was therefore assumed that psychological interventions might be the most logical treatment modality. It now seems highly likely that 'unexplained' regional pain is the result of organic or neurochemical changes; therefore, they are 'medically explained'. Hence, therapeutic modalities that can, even temporarily, reduce neuronal excitability and sympathetic nervous system malfunction may result in just the sort of benefits from local anesthetic blocks documented by Gale et al (pages 185-189). The time is ripe for renewed interest in nerve block models for the relief of pain. Those models are the ultimate foundation of the truly multidisciplinary pain clinic, and their results encouraged pioneers such as Bonica (7) and Travell (8) in taking chronic pain seriously. A look at their work may help to renew some well-established approaches that are currently neglected or out of favour.

\section{REFERENCES}

1. Arner S, Lindblom U, Meyerson BA, Molander C. Prolonged relief of neuralgia after regional anaesthetic blocks. A call for further experimental and systematic clinical studies. Pain 1990;43:287-97.

2. Wall PD. New horizons. An essay. In: Cousins MJ, Bridenbaugh PO, eds. Neural Blockade, 3rd edn. Philadelphia: Lippincott-Raven, 1998: 1135-43.

3. Kim SH, Na HS, Sheen K, Chung JM. Effects of sympathectomy on a rat model of peripheral neuropathy. Pain 1993;55:85-92.

4. McCormack K. Signal transduction in neuropathic pain, with special emphasis on the analgesic role of opioids - Part I: The basic science of phenotype expression in normal and regenerating nerves. Pain Rev 1999;6:3-33

5. McCormack K. Signal transduction in neuropathic pain, with special emphasis on the analgesic role of opioids - Part II: Moving basic science towards a new pharmacotherapy. Pain Rev 1999;6:99-131.

6. Watkins L, Milligan ED, Maier SF. Spinal glia: New players in pain. Pain 2001;93:201-5.

7. Bonica JJ. The Management of Pain. Philadelphia: Lea and Febiger, 1953: 5-7.

8. Travell JG, Simons DG. Myofascial Pain and Dysfunction: The Trigger Point Manual. Baltimore: Williams and Wilkins, 1983.

The views expressed in this editorial are those of the authors and are not intended to reflect the opinions of the Canadian Pain Society or Pulsus Group Inc 


\title{
Anesthésie par blocage nerveux et thérapie cognitivo- comportementale ou leçon d'un « échec »
}

\author{
Harold Merskey DM FRCP, \\ Rédacteur en chef, Pain Research \& Management \\ Ellen Thompson MBBS FRCPC \\ Professeure adjointe d'enseignement clinique, \\ Univerisité d'Ottawa
}

L 'étude réalisée par Gale et coll. (pages 185 à 189) est un exemple utile d'étude non concluante. Contrairement à ceux des études probantes, les résultats d'un échec ne sont guère publiés. Pourtant, on peut aussi en tirer des leçons pour la planification d'études et les traitements.

Pour cette étude, les patients ont été recrutés dans une clinique où on traite la douleur chronique par une série d'anesthésies par blocage nerveux. Les patients avaient le choix de poursuivre leur traitement ou de faire partie d'un groupe de thérapie cognitivo-comportementale. Pour des raisons d'éthique, il leur était permis d'abandonner l'étude sans conséquences. Dès le début, un des 34 patients du groupe d'anesthésie et 12 des 34 patients du groupe de thérapie cognitivo-comportementale ont abandonné. À la fin des huit semaines de l'étude, il restait 33 patients dans le premier groupe et quatre seulement dans le second.

Les auteurs en ont tiré trois conclusions. Premièrement, il ne restait pas suffisamment de patients dans le groupe de thérapie cognitivo-comportementale pour pouvoir comparer l'efficacité des deux démarches. Deuxièmement, il faudra user de prudence dans le recrutement de patients qui manifestent une préférence pour l'un des traitements. Et troisièmement, il faudrait repérer les quelques sujets susceptibles de bénéficier d'une thérapie cognitivo-comportementale afin de les traiter et, si possible, de les suivre.

L'anesthésie par blocage nerveux reste encore une énigme. Ses bienfaits se prolongent au-delà de la durée d'action de l'anesthésique. Ce phénomène documenté est régulièrement observé par les cliniciens, en particulier ceux qui ont une formation en anesthésie. Arner et coll. (1) signalent que, par suite du blocage du nerf afférent, deux tiers des 38 patients souffrant de névralgie ont constaté un soulagement durant parfois jusqu'à six jours. Malgré la popularité de ce traitement pour soulager la douleur chronique, peu d'études ont été faites sur le sujet. Et, bien que l'étude de Gale présente certaines limites, il demeure primordial de poursuivre dans cette voie.

Nul ne sait encore pourquoi les anesthésiques locaux, qui agissent pendant au plus quatre heures contre la douleur aiguë ou " physiologique », soulagent la douleur chronique durant plusieurs jours. Plusieurs théories ont été proposées. Wall (2) en énumère plusieurs dans un texte qu'il a rédigé sur l'avenir des anesthésiques locaux. Le système nerveux sympathique a été mis en cause (3). Dans une série d'articles publiés dans Pain Reviews, McCormack $(4,5)$ avance que le blocage nerveux inhiberait temporairement les décharges ectopiques spontanées, ce qui abolirait l'hyperexcitabilité centrale assurée de manière dynamique tout en accentuant l'inhibition endogène des récepteurs couplés aux protéines $\mathrm{G}$ pour les canaux calciques dépendants du potentiel de type $\mathrm{n}$. Enfin, des données récentes sur l'activation des cellules gliales dans les cas de douleur pathologique (6) pourraient mettre en question l'utilité des thérapies cognitivo-comportementales et autres interventions psy- 
chologiques, et donner une nouvelle justification à l'anesthésie par blocage nerveux, expliquant les résultats de l'étude de Gale et coll. (pages 185 à 189). Les travaux de Linda Watkins et coll. (6) montrent que les cellules gliales de la moelle épinière peuvent être activées en réponse à divers stimuli, comme les lésions tissulaires ou les infections bactériennes et virales. Une fois activées, les cellules gliales produisent un certain nombre de cytokines pro-inflammatoires associées à la sensibilisation centrale. Cette activation se propage de cellule en cellule par les jonctions lacunaires, sans suivre de voies neuronales ou de frontières anatomiques particulières.

Jusqu’à présent "inexpliquée sur le plan médical », la douleur chronique était autrefois désignée par les termes « douleur psychogène », "somatisation » et même " hystérie ». On pensait alors que certains troubles psychologiques d'adaptation étaient la cause première d'une douleur régionale et, par conséquent, qu'une intervention psychologique s'imposait en guise de traitement. Aujourd'hui, il semble très vraisemblable que la douleur régionale « inexpliquée » résulte de changements organiques ou neurochimiques. Elle serait donc « explicable sur le plan médical ». Par conséquent, les modalités de traitement susceptibles, même temporairement, de réduire l'excitabilité des neurones et le dysfonctionnement du système nerveux sympathique offriraient des bienfaits similaires à ceux que procure l'anesthésie par blocage nerveux dont il est question dans l'article de Gale et coll. C'est le moment de se pencher à nouveau sur les modèles de blocage nerveux pour le soulagement de la douleur. Ceux-ci constituent les assises pour l'établissement de cliniques de la douleur vraiment multidisciplinaires et leurs résultats ont incité des pionniers tels que Bonica (7) et Travell (8) à prendre la douleur chronique au sérieux. La lecture de leurs travaux pourrait redonner de l'intérêt à des voies bien établies, mais aujourd'hui négligées ou passées de mode.

\section{RÉFÉRENCES}

1. Arner S, Lindblom U, Meyerson BA, Molander C. Prolonged relief of neuralgia after regional anaesthetic blocks. A call for further experimental and systematic clinical studies. Pain 1990;43:287-97.

2. Wall PD. New horizons. An essay. In: Cousins MJ, Bridenbaugh PO, eds. Neural Blockade, 3rd edn. Philadelphia: Lippincott-Raven, 1998: 1135-43.

3. Kim SH, Na HS, Sheen K, Chung JM. Effects of sympathectomy on a rat model of peripheral neuropathy. Pain 1993;55:85-92.

4. McCormack K. Signal transduction in neuropathic pain, with special emphasis on the analgesic role of opioids - Part I: The basic science of phenotype expression in normal and regenerating nerves. Pain Rev 1999;6:3-33

5. McCormack K. Signal transduction in neuropathic pain, with special emphasis on the analgesic role of opioids - Part II: Moving basic science towards a new pharmacotherapy. Pain Rev 1999;6:99-131.

6. Watkins L, Milligan ED, Maier SF. Spinal glia: New players in pain. Pain 2001;93:201-5.

7. Bonica JJ. The Management of Pain. Philadelphia: Lea and Febiger, 1953: 5-7.

8. Travell JG, Simons DG. Myofascial Pain and Dysfunction: The Trigger Point Manual. Baltimore: Williams and Wilkins, 1983.

Les opinions ici exprimées n'engagent que les auteurs et ne reflètent pas nécessairement celles de la Société canadienne pour le traitement de la douleur ou de Pulsus Group Inc 


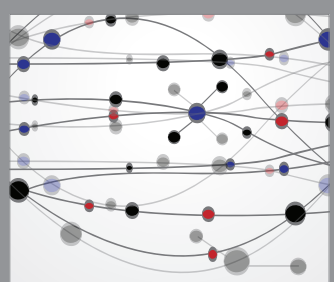

The Scientific World Journal
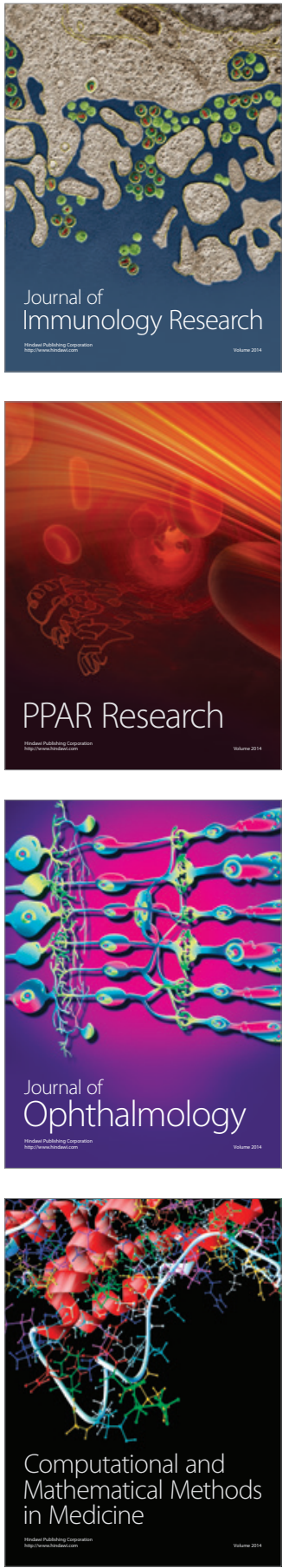

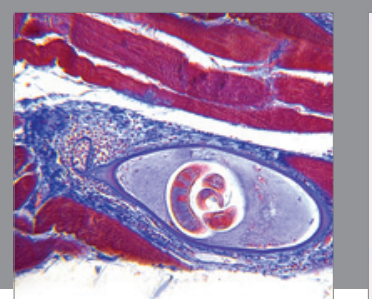

Gastroenterology Research and Practice

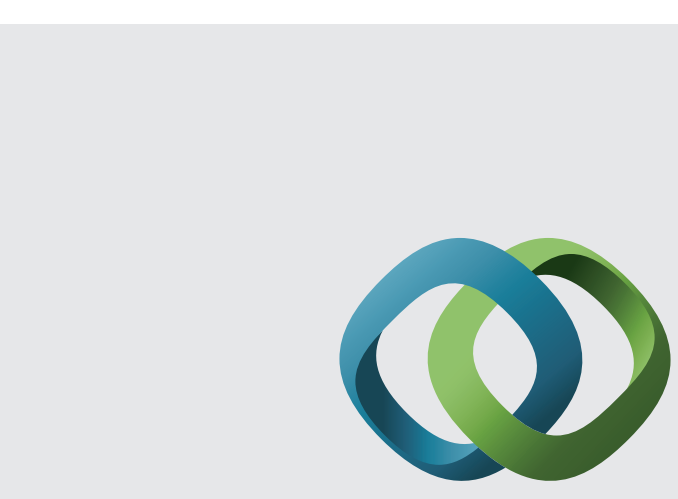

\section{Hindawi}

Submit your manuscripts at

http://www.hindawi.com
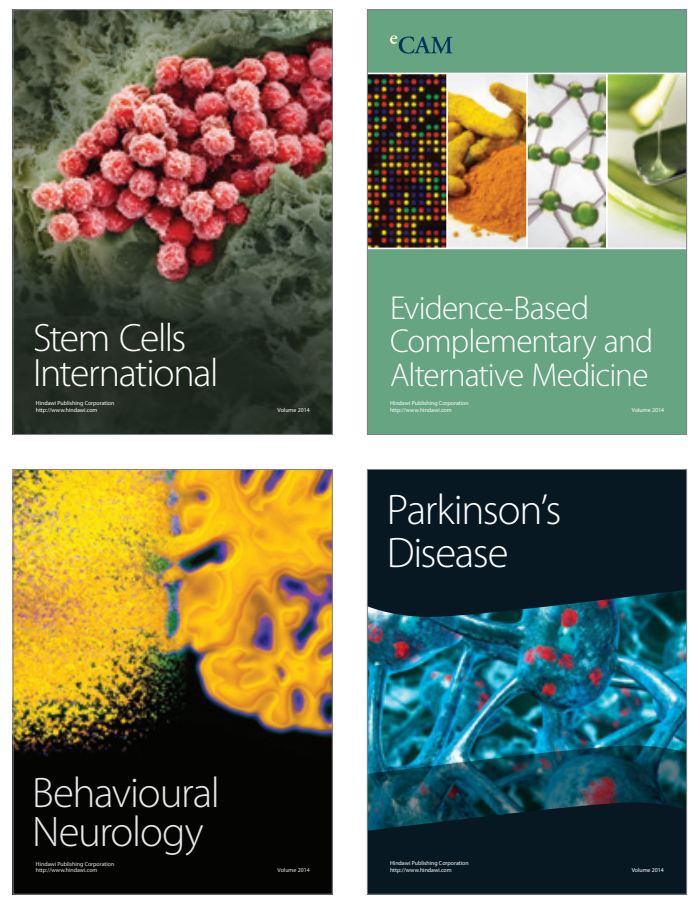
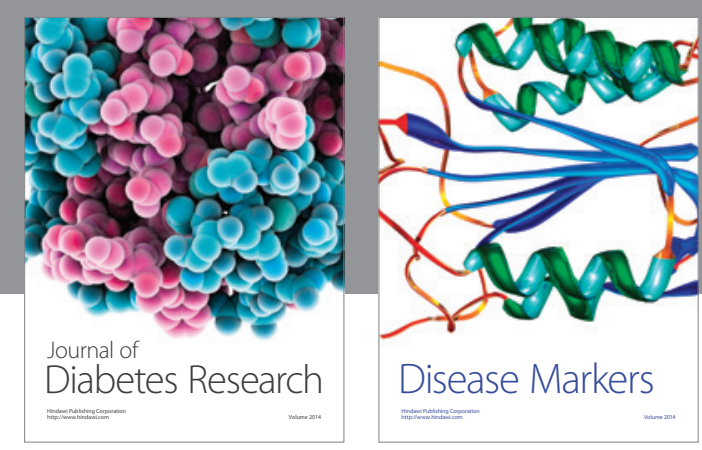

Disease Markers
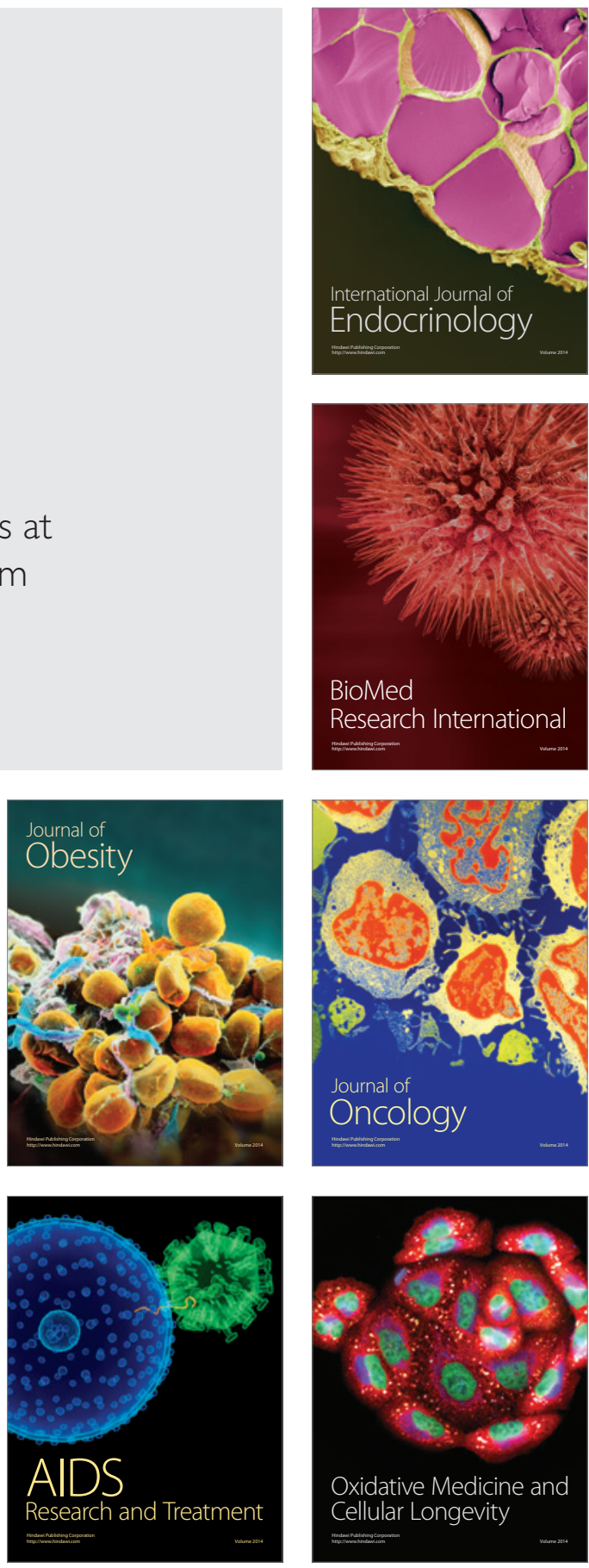